\title{
Safety of Lane Change Maneuver Through A Priori Prediction of Trajectory Using Neural Networks
}

\author{
Ranjeet Singh Tomar and Shekhar Verma \\ Indian Institute of Information Technology \\ Deoghat, Jhalwa, Allahabad-211012 (India) \\ E-mail: rs63@iiita.ac.in, sverma@iiita.ac.in
}

Received: January 5, 2012

DOI: 10.5296/npa.v4i1.1240
Accepted: May 15, $2010 \quad$ Published: May 30, 2010

URL: http://dx.doi.org/10.5296/npa.v4i1.1240

\begin{abstract}
Lane change (LC) is a maneuver that allows drivers to enter into a lane that suits their requirements and comfort. The LC process requires the driver to assess its neighborhood traffic in its original and target lanes before undertaking the maneuver. Other vehicles in the neighborhood also need to adjust for safe lane change. The LC trajectory is determined by the accuracy of these subjective assessments as well as the state of traffic. An erroneous assessment by LC vehicle or neighboring vehicles or an incorrect maneuver can cause collision. The collision can be prevented if the LC trajectory can be predicted and the feasibility of LC can be communicated to different vehicles involved in this process. . In the present paper, neural network is used for long term forecast of the lane change trajectory and for short term near future positions of the LC vehicle. The neural network is trained using past LC trajectories of different vehicles. The trained network is then used for long and short term forecast of the vehicle's positions during LC. Simulation results with actual filed data observed data indicates that neural network is able to learn LC maneuvers and is able to perform short term prediction with sufficient accuracy.
\end{abstract}

Keywords: Lane Change Process, Neural Network, Driver Behavior, Lane Change Trajectory, Traffic Simulation. 


\section{Introduction}

Due to the advances in technology, automobiles are available at cheaper prices leading to increase in the rate of traffic at a much greater pace as compared to the addition of road capacity of the highways [1]. Essential maneuvers like lane change, merging and braking etc in high density scenario cause traffic non equilibrium may also cause accidents, congestion etc. To obviate such conditions, forewarning of such conditions is required. Forewarning systems must be able to predict the onset for such traffic situations or suggest remedial action once the situation has precipitated due to an essential maneuver. Lane change is an essential action by a vehicle which perturbs the traffic and may cause collisions between vehicles. Prediction of the feasibility of a safe lane change and the possibility of collision during lane change is desired for smooth flow of traffic and collision avoidance [2]. Neural network based modeling has attracted increasing attention they are able to learn the traffic conditions and driver behavior at the time of lane change more precisely and realistically than other existing methods [3]. However, the accuracy and validity depends mainly on the drivers' behavior during lane change. Majority of the accidents on highways take place during lane change maneuvers which obstruct the traffic flood and create an overload in addition to loss of precious human life [4], [5]. The advances in technology have made it possible to simulate the complex models of highway traffic and give the warning for safe driving on the highways during the lane changing and overtaking. But some of these models are still inadequate in replicating the real time traffic problem on the highways. These points gives us to the surveillance of the highways traffic and provide the solution for systems that predict the lane change, overtaking, collision avoidance and congestion problems on the highways. We will simulate a neural network based system which dynamically predicts the occurrence of collision and warns the vehicles which are involved in lane change process, so that they can take proper measures such as adjusting their velocities, accelerations by braking or minimum safety distance are taken care of or aborting the lane change, temporarily to avoid the collision and accidents on the highways. Generally in any simulation, traffic models namely car-following and lane changing models are considered to be the part to estimate vehicular movements. Car-following model estimates acceleration and deceleration and positioning of vehicles [6]. Whereas lane change model evaluates lane change decisions on multilane roads based on individual driver goals and purpose. Vehicles often must change lanes, for example, to make a right turn at the next intersection, speed up to pass slower vehicle, slow down to let a faster vehicle pass or to obey transport rules such as lane restriction, priority lanes etc. The vehicular movements cannot be represented accurately in microscopic simulation, if a lane change model is not considered. Lane change models mainly estimates driver lane change decisions, which are usually involved with approximations because of human element. Many lane change models do not considered these approximations and human decisions appropriately in several situations, but assume that drivers estimate current situation and evaluate lane change decisions accurately, which is not the case in reality [7], [8]. In this paper, neural network based modeling has been used to take into account the uncertainties in driver perception in lane change decisions. Several approaches are recently become popular in attempting to overcome these problems include those based on artificial intelligence. In the present paper, our main focus is given to simulate the neural network based modeling of lane 
change process and driver behavior or predict the future of lane change trajectories up to certain interval. Neural network based modeling is not appropriate for expressways only, but also suitable for urban congested traffic conditions. Other appropriate and possible lane change purposes are considered for describe the driver's behavior in urban congested traffic conditions. The comparison of estimated lane change behavior and observed lane change behavior will be confirmed the validity and feasibility of proposed neural network based lane change process and results of the simulation demonstrated the effectiveness of neural network based solutions in lane change process under the lane policy.

The rest of paper is organized as given. Section 2 explains about the related work for safety of lane change through predictions of LC trajectories. Section 3 describes the problem studied in the work. Section 4 discusses lane change phenomenon, briefly discussion on lane change processes and driver's behavior during lane change. Section 5 explains the neural network modeling for lane change maneuver. Section 6 gives the simulation and results based on neural networks for lane changing vehicles during the lane change with input, output variables and associated functions. This section also compares results of the proposed lane change behavior to observed lane change behavior on the highways for validation purpose and assesses the impact of neural network based solutions in the lane change process according to lane policy. Section 7 presents the conclusion of the work.

\section{Related Work}

Prediction of vehicular motion and driving behaviors has been given considerable attention in the development of vehicle active safety system. A vehicle active safety system must give meaningful and trust worthy information to enhance the safety of the transportation system. Therefore, it is important to integrate driving behavior models and trajectory prediction techniques into system designs to decrease driver's burden and enhance driving safety. Gap acceptance theory [9] has been used for the representation of the driver's behavior and prediction of safe trajectories of LC vehicles before the initiation of the lane change process. In [10], authors propose minimizing overall braking induced by lane change to derive lane change rules. Hidden markov models (HMM) have been employed in [11] to recognize the lane change state and trajectory of the vehicles. In this work, different features have been identified to classify lane keeping and lane changing behaviors. SVM and model tracking have been used in [12] to increase the performance of lane change classifiers for forecasting of the vehicular trajectory. In [13], sensors have been deployed for sensing the latitudinal position and head motion to infer lane change intent. This study shows the possibility of predicting lane change trajectories and possible collisions before lane change maneuvers are initiated using sensors onboard and inside the vehicle. This method is able recognize the stages of lane change process, as well as provide the information about the start and end of lane change maneuver. However, the method is not able perform long term prediction of lane change trajectories. In [14], authors develop an HMM with a set of dynamic models to recognize vehicle motion, driver behavior and predict drivers' subsequent actions. Cognitive architectures have been proposed in [15] to analyze the relationship 
between the perceived visual factors and steering angle of lane change behavior for trajectory prediction. A piecewise polynomial model has been used to represent different stages of lane change maneuver with varying regression models and analyze lane changing behavior of different drivers and LC vehicles [16]. However, the coefficients vary with change in drivers and the LC scenarios that are difficult to determine beforehand. A stochastic switched autoregressive exogenous model for modeling and recognition of lane change maneuver of different LC vehicles and prediction of LC trajectory has been proposed in [17]. In [18], the driver steering action is modeled based on the target headway error for double lane change. However, the preview destination points and vehicle current heading angle are needed to compute the destination headway error and forecast the safe trajectories. In driver assistance systems, dynamic model can be used to plan safe and feasible lane change trajectories more efficiently as compared to other models [19]. In [20], different models have been proposed for generating ideal lane change trajectory prediction. In [21], the lane change trajectory has been modeled as a sinusoidal function. This model has extensively used [21]. In [22], sinusoidal and trapezoidal functions are used to describe lane change maneuvers for analyzing lane change crashes. However, fitting the restrictions of lane change behavior by adjusting model parameters is difficult and makes the technique infeasible for prediction of trajectories. In [23], the lane change trajectory has been generated using a fifth order polynomial. However, the method is able to generate a smooth lane change path only when the initial and final vehicle states are fixed. Therefore, it does not correspond to actual lane change trajectories and the method cannot be used for predicting impending collisions on the road. In [24], authors propose a set of dynamic conditions of safe and unsafe lane change trajectories between related vehicles during lane change maneuvers. The lane change trajectory is generated according to a sinusoidal function. Different longitudinal acceleration models have been examined in [25] to determine safe and unsafe regions, as well as the minimum longitudinal safe spacing between vehicles and surrounding vehicles for lane change scenarios. In [26], according to relative longitudinal distance and speed, four states have been used to efficiently label the severity level of lane change behavior. In [27], different vehicle sensors have been used to detect the driving environment and assess the safety of lane change maneuvers based on a Bayesian network. In [28], Monte Carlo sampling is applied to compute the collision probability and forecast the safe vehicular trajectories during lane changing. The method can simulate possible lane changing traffic behaviors and assess safe regions with collision probability. However, because of the countless options and improper prior distribution of the input parameters, the collision probability results sometimes considerably deviate from the appropriate forecast values. Therefore, the efficient generation of different types of trajectories and time to collision and minimum gaps by this model will be highly useful for predicting potential collisions in transportation systems. The work in [29] focuses on modeling lane change behavior as a dynamic model for safe and feasible lane change trajectories between LC vehicle and other participating LC vehicles. This model clearly describing different driving behaviors and but cannot efficiently forecasting the lane change trajectories and predict the impending collisions on the road. Moreover, the lane change dynamic model is not used to autonomously and smoothly control a vehicle as it changes lanes. This lane change model 
also reflects the driver control strategy of adjusting longitudinal and latitudinal acceleration, compute the time to collision and minimum acceptable gaps for every lane change trajectory to identify the safe lane change trajectories and ensure the transportation safety to some extents.

\section{Problem Description}

The lane changing maneuver is one of the critical actions that a driver performs while travelling on a highway. Drivers perform some action and reaction during driving on the highways according to his destination or time dependent events. This reaction is perceived by the following vehicle and this continues. The perception-reaction time constitute the propagation delay. In critical cases, propagation delay may not leave sufficient maneuvering time to avoid collisions if inter-vehicles spacing is not adequate. Errors in the lane changing decision-making process, typically caused by driver failure to accurately and timely interpret information about other vehicles in close proximity, have often resulted in serious accidents. In order to eliminate such errors, or at least minimize their impact, and increase the level of safety, the vehicles of the future would have to incorporate intelligent solution that will allow them to accurately consider all aspects of a lane-changing maneuver. For this, a number of real-time parameters need to be considered and estimated; First, acquiring parameters like velocity, acceleration, deceleration and position of neighboring vehicles; second, determining the start of lane change maneuver; and third, estimating optimal and safe trajectories, and minimum safety distance between the vehicles. Neural network solution promise to provision functionalities like vehicular safety and traffic stability on the highways through lane change trajectory prediction. Vehicles and drivers share this information with each other for collision prevention and safe driving on the road.

\section{Lane Change Phenomenon}

\subsection{Responsible and Safe Lane Changing}

Vehicles need to changing lanes on highways with more than one lane in the same direction. Vehicles also want to change lanes to overtake another vehicle, simple lane changing for fast movement, to avoid parked vehicles in the lane or when the vehicle ahead slows/fast to turn at an intersection of the lanes. Vehicles provide always proper information before changing lanes and ensure the movement can be made safely. Vehicles cannot change lanes suddenly by cutting in front of another vehicle on the road. The other vehicles will not be expecting your maneuver or behavior on the road. Vehicles always avoid changing lanes unless required. Vehicles cannot change lanes in or near an intersection of the lanes. Spending a few seconds behind another vehicle is often safer than going around it.

\subsection{Steps for Lane Changing}

- Check for a space in lanes where you can move safely. 
- Give information intelligently in the direction you want to move or lane change on the highways.

- Ensure the way is clear and that no one is coming at a fast speed from behind. Maintained safety distance from other vehicles.

- Turn steadily into the new lane.

\subsection{Lane Change Process}

The Neural network based model predicts the occurrence of collision during the lane change on the highways. Vehicles consider the characteristic variables during lane change such as the relative velocity of the vehicles, time taken by the vehicle to make the lane change on the highways, minimum safety distance, acceleration or deceleration of the both the vehicles, etc. Considering the above parameters, derive set of equations for the calculation of the lane change criteria and taken into consideration to predict the safe lane changing on the highways. Based on the above rules estimate whether a successful lane change will occur, collision will take place or an undesired lane change will occur. Before collision vehicle will provide warning alarm of danger state to the other vehicles that change the lane on the highways. Now based on the above set of possibilities calculate the mean square error and cross validation of mean square error of the occurrence of each of the possibilities like collision, near collision, absolute safe and safe condition on the road during lane changing. These calculations are used to train, test and production of the real data of vehicles those involved in the lane change process to predict the occurrence of the above scenarios. For development of proper lane change systems on the highways, it is necessary to know the dynamic conditions of the systems that characterize the accidents during a lane change. For simplicity, consider only three vehicles each in a different lane on the highways. Study the different ways in which the lane changing can take place and the necessary safety steps or conditions to be followed. Sub divides these conditions based on their velocities, acceleration and deceleration. This phenomenon can be extend the principles used in calculating of safe minimum distance and the estimation of lane change to multiple vehicles and multiple lane changes on the highways. The same analysis can be extended for anomalous lane changes (lane change on curves) on the highways. Considering other factors such as acceleration of the vehicles, friction of the highways road, friction provided by the tires of the vehicles and so on we can predict more accurately the occurrence of collision and analyzed the lane change process more accurately using neural network modeling. Use of actual lane change data for modeling the system will aid us in generating a more appropriate model for estimating the process of lane change on the highways for collision prevention. We also consider the reaction time of the drivers or vehicles for the lane changes on the highways. The minimum safety distance will depend on the reaction time of the drivers or vehicles at the time of lane changes on the highways [8], [30] and [31].

\subsection{Driver Behavior during Lane Change Process}

A driver plans his journey from the source to his final destination and estimates the time needed to reach the final destination. Accordingly, he starts his journey from the source to reach the final destination as per time assigned [31], [32]. On the highway, the normal driver 
may face different traffic states like congestion, traffic signal and slow ahead vehicles with his non lane changing attitudes, which may affect his original travel-time estimation. To keep this estimation, the behavior of the driver may change from normal behavior to aggressive behavior. He may ignore the safety rules and minimum gap with the preceding vehicle, change lanes aggressively fast. He may also be prone to driver errors on the highways. When driver has not keeps the patience during lane changing, so his behavior changes from his normal behavior to a more aggressive behavior which may progressively aggravate. The aggressive behavior of the driver is characterized by increased speed, reduced time gap, and rapid acceleration and deceleration on the highways. This aggressive behavior also increases driver indiscretion and risk taking nature on the road. The change in behavior coupled with the lack of the awareness of the global scenario of the traffic state for spatial temporal anticipation and reduced maneuvering time may result in collision when the front or side ahead vehicle brakes or during lane changing on the highways. Absolute errors in the decision-making time typically caused by driver at the time of lane change failure to accurately and timely interpret information about other vehicles in close proximity, have often resulted in serious accidents and congestion on the highways [32], [33].

\section{Neural Network Modeling}

\subsection{Lane Change Maneuver}

Lane change is a frequent action undertaken by vehicles on the road and usually results in a definite gain to the vehicle in terms of driving comfort or reduced time to travel. Lane change can be defined as an intentional and significant shift in the lateral position of a vehicle from one lane to another (Figure 1). Lane changing vehicles moving on the road form a travelling path like a-b-c-d-e-f-g as shown in Figure 1. Continual change in lateral positions within a lane or an unintentional foray into an adjacent lane by a meandering vehicle does not constitute a lane change. It could be a deliberate voluntary action, a mandatory procedure in a merging / splitting section of the road or an act forced by a slower leading vehicle or a faster following vehicle. The process itself is risky and is performed voluntarily only when the gain offsets the risk. The lane change process can be viewed as a series of multiple distinct phases: Planning phase, Preparation phase, Crossover phase and the Adjustment phase. Whenever the driver intending a lane change finds a sufficient gap between vehicles in the target lane, the planning phase of lane change commences. The planning phase is devoid of any physical intervention. In this phase, a driver assesses the feasibility of lane change and also estimates the benefit of a lane change vis-a-vis the risk involved in the process. This assessment is subjective and depends on the nature of the driver, his assessment of the behavior of drivers in his target lane, the relative position and speed estimates and its predicted speed and longitudinal and lateral acceleration / deceleration required for a safe lane change in addition to the gain to be derived from lane change. If the driver decides to change the lane, the preparation phase starts. In this phase, the driver continues in his trajectory in the initial lane but accelerates or decelerates relative to the projected position of the vehicles in the destination lane just after its entry into the lane. After this adjustment, the vehicle accelerates 
laterally to shift in the chosen destination lane. The crossover phase is characterized by significant initial acceleration and then a deceleration after the vehicle has crossed over to the destination lane. This lateral acceleration should be less than the comfortable acceleration threshold and the lateral speed is greater than zero and less than the maximum permissible lateral speed. The longitudinal speed is usually assumed to be constant. However, actual lane change data shows that the speed varies during the crossover phase. Around $80 \%$ of the drivers accelerate / decelerate during the crossover phase. Of this $60 \%$ accelerate while others decelerate to achieve safety in the target lane. This ensures a smooth curvature of the vehicle trajectory and allows safe braking in the event of lane change abortion. In the last phase, the vehicle accelerates/decelerates to adjust its speed and to maintain a safe gap in accordance with the state of its leading vehicle. Thus, the lane change process can be characterized by variable speeds with constant acceleration and deceleration in both longitudinal and lateral directions.

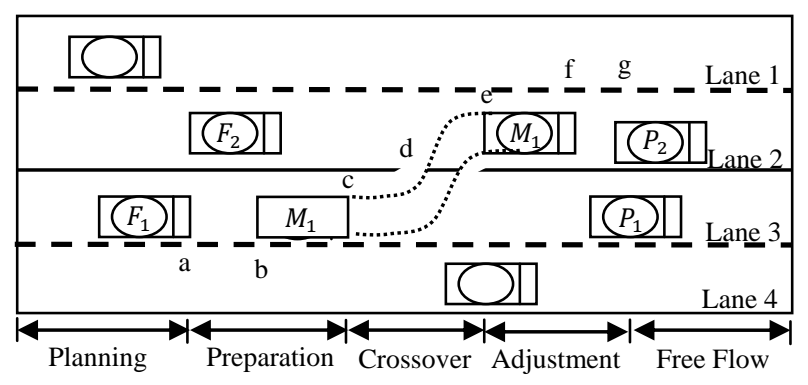

Figure 1. Lane Change Maneuver

\subsection{Neural Network Approach}

Neural networks are non-linear statistical data modeling tools. They can be used to model complex relationships between inputs and outputs or to find output patterns in data. Neural network approach offer a potential alternative to other methods like HMM (Hidden Markov Model) based techniques for prediction of lane change within road traffic scenarios. This paper proposes neural network based modeling for lane changing decisions of drivers. Two approaches are considered. The first, preliminary approach uses a neural network modeling with a single hidden layer and the back propagation learning algorithm to model lane change process and predict the trajectory of lane changing vehicles. A series of consecutive time space traffic patterns, which describe the lane change initiation and changes in the trajectories over time by the vehicle, are input to the neural network, which then predicts the target lane and position of the vehicle. Real time data from NGSIM Community have been used for neural network modeling, training, testing, production and validation. The trained neural network successfully exhibited the rudiments of lane and speed changes. The second approach concentrates specifically on lane changing and makes use of a learning classification type of neural network. Input to the neural network still consists primarily of time space traffic patterns. The neural network output classifies the input data by determining the new lane for the vehicle concerned. Performance metrics in above two approaches i. e. testing, training, production and validation were acceptable for data generated by the rule 
based lane change model of a neural network simulation. Performance in testing was less satisfactory for data taken directly from the road. This highlighted the need for extensive data sets for successful training and also emphasized the need for data cleaning and preprocessing to overcome the inaccuracies of data acquisition [34].

\subsection{Neural Network Based Modeling}

Neural network based modeling can be used to model and test the time dependent real data. To model the lane change real data we used a single or two hidden layers MLP model with 8 inputs. Perhaps the results could have been improved by using more inputs. This modeling technique will show the test and production process to evaluate the performance of a neural network trained time dependent real data of the traffic highways. This model predicting the lane change behavior in advance up to some extent satisfactorily. The inputs to the model consist of the relative velocity of the vehicles who involved in the lane change and travel in the passing lane, acceleration / deceleration, safety distance between the vehicles, and current states of the vehicles in which vehicles are travel (collision, near collision, absolute safe and safe) on the highways. The output of this model is the prediction of the next states during the lane change on the highways. We are using the real time data of the highways which cover the velocity, acceleration / deceleration and safety distance range throughout the lane change on the highways. We will test the neural networks performance of the real data. Since, this is a real lane change modeling application, so the true value of this modeling can only be determined by applying the model to a lane change strategies and computing the safety profits over several time spans. An algorithm shows the selection of the number of hidden neurons in the neural network modeling. The optimal construction of back propagation (BP) ANN is a four step process as described below (Figure 2):

\section{Step 1: Input and Output:}

The inputs to the system are the position, velocity and acceleration / deceleration of the lane change vehicle with future position values as output.

Inputs:

i. The longitudinal positions of the lane changing vehicle.

ii. The lateral positions of the lane changing vehicle.

iii. The velocity of lane changing vehicle.

iv. The acceleration / deceleration of lane changing vehicle.

v. The longitudinal positions of the following / preceding vehicle in destination lane.

vi. The lateral positions of the following / preceding vehicle in destination lane.

vii. The velocity of following / preceding vehicle in destination lane.

viii. The acceleration / deceleration of following / preceding vehicle in destination lane.

Outputs:

i. The longitudinal positions change of the lane changing vehicle.

ii. The lateral positions change of the lane changing vehicle. 
The accuracy of prediction may improve with these inputs and our aim is to study the practicability of short range and long range trajectory prediction only from the past values of position and velocity of the trajectory of lane changing vehicle $\left(V_{L C}\right)$ itself and destination lane following vehicle $\left(F_{d}\right)$. No underlying model or statistics of the states or driver characteristics is considered. The $V_{L C}$ and $F_{d}$ time series can be modeled using AR representations. The bivariate linear prediction model for $V_{L C}$ and $F_{d}$ is

$$
x_{L C}(t)=\sum_{i=1}^{\infty} a_{11} x_{L C}(t-i)+\sum_{i=1}^{\infty} a_{12} x_{F}(t-i)+e_{12}(t)
$$

Where $X_{L C}: x_{L C}(1), \ldots \ldots \ldots$ and $X_{F}: x_{F}(1), \ldots \ldots \ldots$ are stationary stochastic processes and $e_{12}(t)$ is the error. The bivariate time series consider the causal influence of $X_{F}$ and the stochastic process $X_{L C}$.

\section{Step 2: Neural Network Creation:}

The neural network based model creates an interconnection between the processing elements (PEs) in the form of weighted matrix populated by the time series of the input parameters. In this recurrent network, some of the connections may be absent, but there are feedback connections. An input presented to a recurrent network at time $t$ will affect the networks output for future time steps greater than $t$. Therefore, a recurrent network needs to be operated over time.

\section{Step 3: Training and Testing:}

The training and testing set is used to test the performance of the network. Once the network is trained the weights are frozen, the testing set is fed into the network and the network output is compared with the desired output. The weights are changed based on their previous value and a correction terms and rules. Once the rule is selected, correction should be applied to the weights, as per the learning rate. If the learning rate is too small, then learning takes a long time. If it is set too high, then the adaptation diverges and the weights are unusable. For the momentum component there are two parameters to be selected: the step size and the momentum. We determine the best values for these parameters and for optimized modeling require that the network be trained multiple times in order to produce the lowest error. This model terminates the training based on mean squared error. The square error function in the prediction is

$$
S E(k)=\frac{1}{2}[\hat{f}(t+k)-f(t+k)]^{2}
$$

Where $S E(k)$ - Error function, $\hat{f}(t+k)$ is the forecasted value and $f(t+k)$ is the real observed value. The training terminates when the mean square error (MSE) drops below the specified Threshold. Cross validation monitors the error on an independent set of data and stops training when this error begins to increase. We use the threshold value for this model is 0.0001 .

\section{Step 4: Production and Errors:}


The most important factors for the evaluation of a neural network is the production and the testing error. Production involves predicting the value of a variable from a series of observations of input variables up to certain time. The observations $\{o(T), \ldots \ldots o[t-$ $N$-1 zavailable at discrete and predefined time interval $\tau\}$ constitute a window. This observation window is used for prediction. A window size that is too small will not allow the model to properly represent the system dynamics, and will therefore lead to a poor generalization performance. Conversely, if the window is too large then the computational complexity of the network is unnecessarily increased. Moreover the network may also learn to represent both the signal and noise in the data which will again lead to poor generalization. The production aim is to predict the value $\hat{o}_{p}(T+L)$, where $L$ is the lead-time and is an integral multiple of $\tau \square$ Here, a sliding window consisting of 30 observations have been taken for prediction of 10 future position values. More formally the production objective is to obtain a prediction function $o_{p}(T+L)$ which minimizes the mean square of the deviations $o_{p}(T+L)-o(T+L)$ for each lead time $L$. Training error must be extremely low. If the training error is low 0.0001 , the production of the desired output efficiently approaches to the realistic values. Testing error actually evaluates the neural network performance and the better production of the real data.

\section{Algorithm for selection of the number of hidden neurons:}

Step 1: Select a small number of hidden neurons in layer.

Step 2: Train the neural network and evaluate the performance of neural network.

Step 3: Check the acceptable momentum value of neural network.

Step 4: If acceptable momentum value will not achieved then again train the neural network and evaluate the performance of neural network.

Step 5: If acceptable momentum value will achieved then check the acceptable mean square error value of neural network.

Step 6: If acceptable mean square error value will not achieved then again select a small number of hidden neurons in the layer.

Step 7: If acceptable mean square error value will achieved then the process of selection of small number of hidden neurons in the layer will stop. 


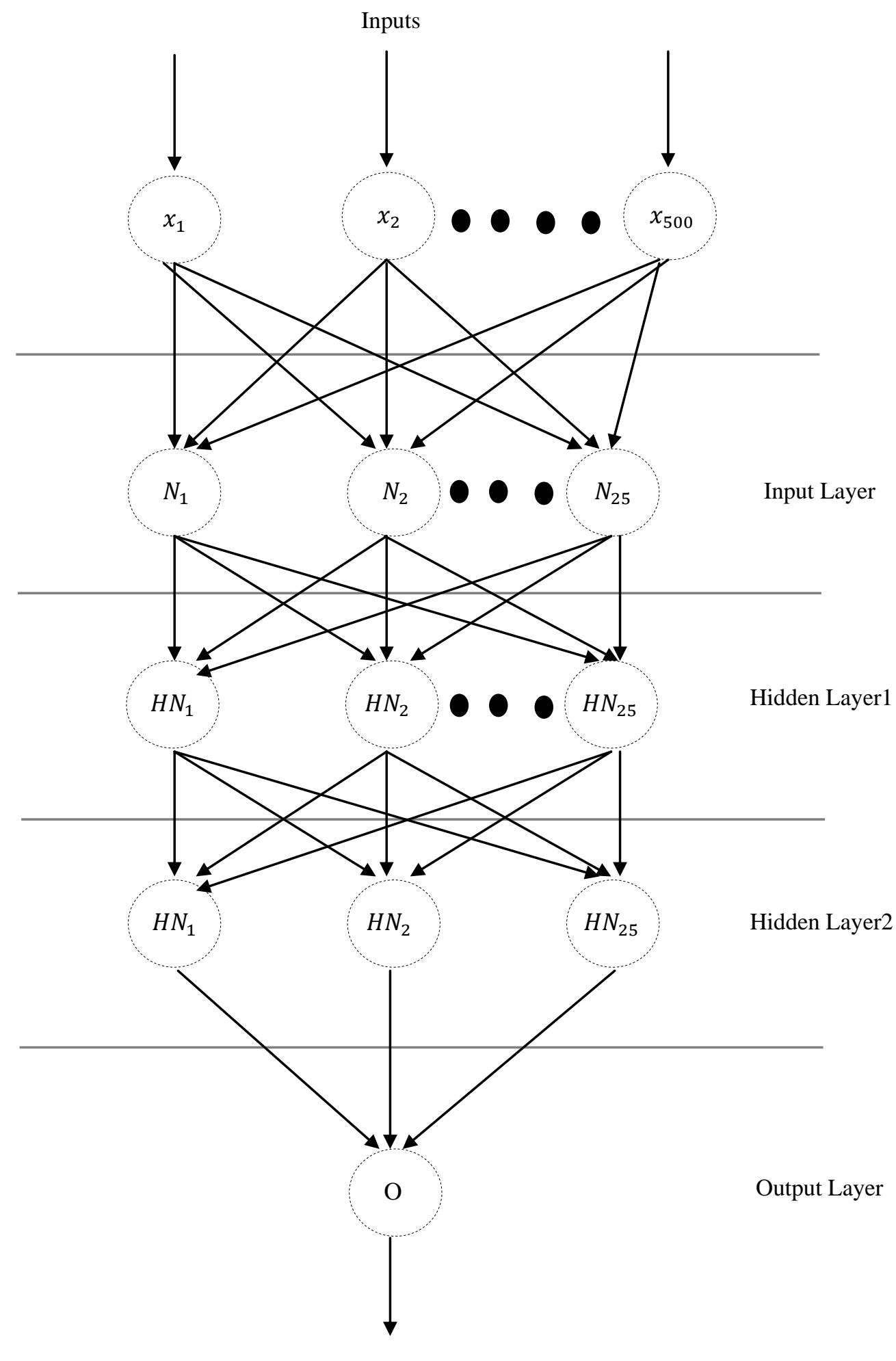

Output

Figure 2. Neural Network with Two Hidden Layers 


\section{Simulation and Results}

Next Generation Simulation (NGSIM) data provide a unique opportunity to investigate driver behavior, better understand traffic dynamics and improved lane change models. The NGSIM freeway database consists of vehicle trajectories on two test sites. The I-80 (BHL) test section is a 0.40 mile $(0.64 \mathrm{~km})$ 6-lane freeway weaving section with an HOV lane. Processed data include 45 minutes of vehicle trajectories in transition (4:00-4:15 pm) and congestion (5:00-5:30 pm). The US101 site is a 0.3 mile $(0.5 \mathrm{~km})$ weaving section with five lanes. Processed data include 45 minutes of vehicle trajectories in transition (7:50-8:05 am) and congestion (8:05-8:35 am). The data have been extracted from video recordings using machine vision algorithms. The work is part of an ongoing experimental study on empirical understanding of traffic dynamics and formulation of improved models for the traffic highways [35], [36]. The methods here is generic and can be used for prediction analysis based on vehicle trajectories estimated from any range sensors as long as the speed trajectories are reasonably smooth. The NGSIM data format is vehicle ID, lane and position at $0.1 \mathrm{sec}$ intervals. We processed the data to produce time distance and time speed format for each freeway lane, and also speed distance formats for each lane. A total of 200 vehicles were processed. In which we consider 100 vehicles were involved in the lane change. Some of the vehicles were involved in the multiple lane change but we consider only single lane change. Available real time data is in raw form. It is processed and smoothed before usage.

Using neural network based modeling, we train a time dependent real traffic data to classify and predict the lane change process either collision, near collision, absolute safe and safe states on the road. Cross validation is required for preventing over-training. The Figure 1 shows the learning curves for both the real traffic data training and cross validation of real traffic data sets. The actual learning curves will vary with each time points and approaches to zero for the better training of the real traffic data. All training processes the minimum cross validation error (when cross validation is used) or at the minimum training error (when cross validation is not used). For better modeling of the real traffic data, the network must be trained multiple times. $\mathrm{N}$ times training procedure provides the statistical validity of a model. This can be seen by the results that the standard deviation decreases as the final epoch is approached.

We consider 180 time point from the real time traffic data. The velocity of lane change vehicle, velocity of passing lane vehicle, average acceleration / deceleration and safety distance will serve as inputs to the neural network modeling and state (collision, near collision, absolute safe and safe states) will serve as the outputs of the neural network modeling. The data has been pre-sampled such that the first 60 time points contain the data of vehicle and vehicle velocity varies from $8 \mathrm{~m} / \mathrm{s}$ to $30 \mathrm{~m} / \mathrm{s}$ for the training, the next 60 time points contain the data of vehicle and vehicle velocity varies from $8 \mathrm{~m} / \mathrm{s}$ to $30 \mathrm{~m} / \mathrm{s}$ for the testing, and last 60 time points contain the data of vehicle and vehicle velocity varies from 8 $\mathrm{m} / \mathrm{s}$ to $30 \mathrm{~m} / \mathrm{s}$ for the production of the lane change trajectory of the vehicle. In our problem, the back propagation neural network with single hidden layers is selected. A single hidden layer MLP has been pre-trained on this data and the neural network modeling results are shown in the Figure 4 and Figure 5. Figure 4 and Figure 5 shows the actual trajectory verses predicted trajectory of vehicle in safe vehicle condition in the longitudinal direction as 
well as in the lateral direction.

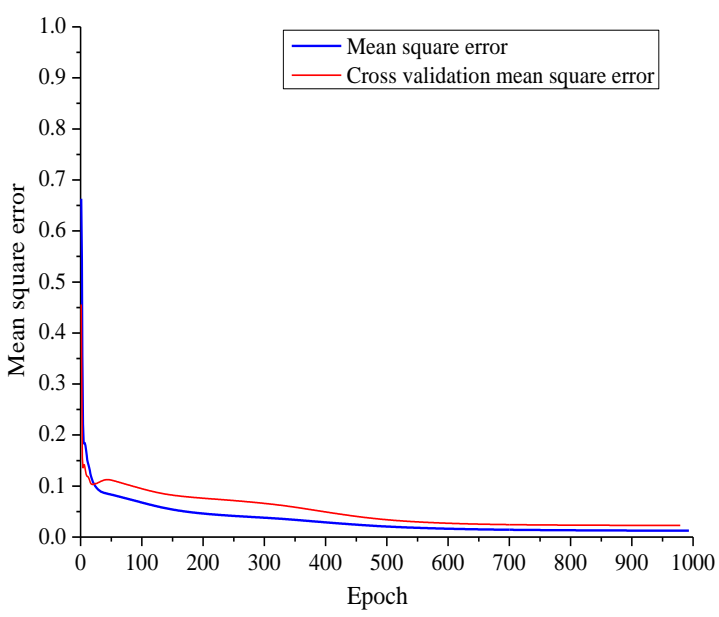

Figure 3. Learning Curve of Mean Square Error $v / s$ Cross Validation of Mean Square Error

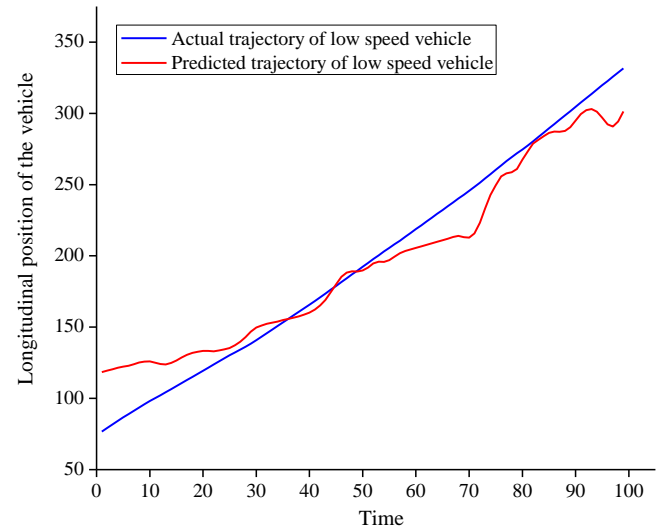

Figure 4. Actual $v / s$ Predicted Trajectory of Vehicle in Safe Vehicle Condition in Longitudinal Direction

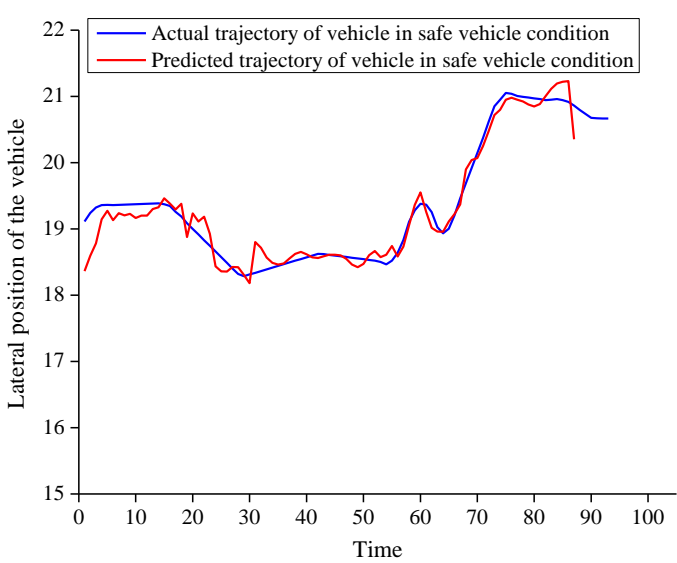

Figure 5. Actual $v / s$ Predicted Trajectory of Vehicle in Safe Vehicle Condition in Lateral Direction 


\section{Conclusion}

A Sensor system on a car can continually observe and measure a multitude of parameters like coordinates of cars, their velocities, accelerations, inter vehicle distance etc. These parameters can be used to predict the future positions of a vehicle during lane change. The lane change trajectory is also affected by the nature of the driver and their assessment of the traffic state of the neighborhood. These subjective inputs significantly affect the lane change trajectory and also render modeling techniques inaccurate. A neural network is able to imbibe the subjective influence on the LC trajectories and predict both the short and long term future positions of the vehicle with sufficient accuracy. Forecast of the future trajectory can be used to forewarn a vehicle to undertake the lane change or abort it depending on the amount of the risk of collision. Short term prediction can be used by the vehicle to adjust to some unexpected change in the neighborhood traffic.

\section{References}

[1] Malta, L., Miyajima, C. and Takeda, K., "A Study of Driver Behavior under Potential Threats in Vehicle Traffic", In IEEE Transactions on Intelligent Transportation Systems, vol.10, no. 2, 2009, pp. 201-210. http://dx.doi.org/10.1109/TITS.2009.2018321

[2] Tomar, Ranjeet Singh and Verma, Shekhar, "Neural Network Based Lane Change Trajectory Prediction in Autonomous Vehicles", In Springer Transactions on Computational Science, vol. 13, LNCS 6750, 2011, pp. 125-146. http://dx.doi.org/10.1007/978-3-642-22619-9_7

[3] Tomar, Ranjeet Singh, Verma, Shekhar and Tomar, G. S., "Prediction of Lane Change Trajectories through Neural Network", In IEEE International Conference on Computational Intelligence and Communication Networks, 2010, pp. 249-253. http://dx.doi.org/10.1109/CICN.2010.59

[4] Chaurasia, B. K. and Verma, S., "Haste Induced Behavior and VANET Communication", In IEEE International Conference on Vehicular Electronics and Safety, 2009, pp. 19-24. http://dx.doi.org/10.1109/ICVES.2009.5400328

[5] Ortiz, M.G., Fritsch, J., Kummert, F., Gepperth, A., "Behavior Prediction at Multiple Time-Scales in Inner-City Scenarios”, In IEEE Intelligent Vehicles Symposium (IV), 2011, pp. 1068-1073. http://dx.doi.org/10.1109/IVS.2011.5940524

[6] Kesting, A., Treiber, M. and Helbing, D., "General Lane-Changing Model MOBIL for Car-Following Models", In Journal of the Transportation Research Board, No. 1999, Transportation Research Board of the National Academies, Washington, D.C., 2007, pp. 86-94. http://dx.doi.org/10.3141/1999-10

[7] Jula, H., Kosmatopoulos, Elias B. and Ioannou, Petros A., "Collision Avoidance Analysis for Lane Changing and Merging", In IEEE Transaction on Vehicular Technology, vol. 49, no. 6, 2000, pp. 2295-2308. http://dx.doi.org/10.1109/25.901899

[8] Caveney, D., "Cooperative Vehicular Safety Applications", In IEEE Control System Magazine, vol. 30, issue 4, 2010, pp. 38-53. http://dx.doi.org/MCS.2010.937003

[9] Gipps, P.G., "A Model for the Structure of Lane Changing Decisions", Transportation Research Part B: Methodological, Elsevier, vol.20, no.5, 1986, pp.403-414. http://dx.doi.org/10.1016/0191-2615 (86)90012-3 
[10]Bishop, R. "Intelligent Vehicle Technology and Trends", Artech House, 2006, pp. 50-80.

[11] Oliver, N. and Pentland, A. P., "Graphical Models for Driver Behavior Recognition in a SmartCar", Proceedings of the IEEE International Symposium on Intelligent Vehicles, 2000, pp.7-12. http://dx.doi.org/10.1109/IVS.2000.898310

[12]Mandalia, M. H. and Salvucci, D. D., "Using Support Vector Machine for Lane-Change Detection", Proceedings of the 49th Human Factors and Ergonomics Society Annual Meeting, vol.49, no.22, 2005, pp.1965-1969. http://dx.doi.org/10.1177/154193120504902217

[13] McCall, J. C., Wipf, D. P., Trivedi, M. M. and Rao, B. D., " Lane Change Intent Analysis using Robust Operators and Sparse Bayesian Learning", Transactions on Intelligent Transportation Systems, IEEE, vol.8, no.3, 2007, pp. 431-440. http://dx.doi.org/10.1109/TITS.2007.902640

[14]Pentland, A. and Liu, A., "Modeling and Prediction of Human Behavior", Journal of Neural Computation, vol.11, no.1, 1999, pp. 229-242. http://dx.doi.org/10.1162/089976699300016890

[15] Salvucci, D. D., "Modeling Driver Behavior in a Cognitive Architecture", Journal of the Human Factors and Ergonomics Society, vol.48, no.2, 2006, pp.362-380. http://dx.doi.org/10.1.1.72.8492

[16]Kim, J. H., Hayakawa, S., Suzuki, T., Hayashi, K., Okuma, S., Tsuchida, N., Shimizu, M. and Kido, S., "Modeling of Driver's Collision Avoidance Maneuver Based on Controller Switching Model", Transactions on Systems, Man and Cybernetics, vol.35, no.6, 2005, pp.1131-1143. http://dx.doi.org/10.1109/TSMCB.2005.850168

[17]Sekizawa, S., Inagaki, S., Suzuki, T., Hayakawa, S., Tsuchida, N., Tsuda, T. and Fujinami, H., "Modeling and Recognition of Driving Behavior Based on Stochastic Switched ARX model", Transactions on Intelligent Transportation Systems, IEEE, vol. 8, no.4, 2007, pp.593-606. http://dx.doi.org/10.1109/TITS.2007.903441

[18]Tan, H. S. and Huang, J. H., "Experimental Development of a New Target and Control Driver Steering Model Based on DLC Test Data", Transactions on Intelligent Transportation Systems, IEEE, vol.13, no.1, 2012, pp. 375-384. http://dx.doi.org/10.1109/TITS.2011.2174785

[19]Enke, K., "Possibilities for Improving Safety within the Driver Vehicle Environment Control Loop", Proceedings of the $7^{\text {th }}$ International Technological Conference on Experimental Safety Vehicles, 1979, pp.789-802.

[20]Nelson, W., "Continuous-Curvature Paths for Autonomous Vehicles", Proceedings of the IEEE International Conference on Robotics and Automation, 1989, pp. 1260-1264. http://dx.doi.org/10.1109/ROBOT.1989.100153

[21]Papadimitriou, I. and Tomizuka, M., "Fast Lane Changing Computations using Polynomials", Proceedings of the IEEE International Conference on American Control Conference, vol. 1, 2003, pp. 48-53. http://dx.doi.org/10.1109/ACC.2003.1238912

[22]Bascunana, J. L., "Analysis of Lane-Change Crash Avoidance”, Society of Automotive Engineers- Technical Report, Vol.951895, 1995, pp. 33-41.

[23] Miyajima, C., Nishiwaki, Y., Ozawa, K., Wakita, T., Itou, K. and Takeda, K., "Cepstral Analysis of Driving Behavioral Signals for Driver Identification”, Proceedings of the 
IEEE International Conference on Acoustics, Speech and Signal Processing, vol.5, 2006, pp.921-924. http://dx.doi.org/10.1109/ICASSP.2006.1661427

[24] Schubert, R., Schulze, K. and Wanielik, G., "Situation Assessment for Automatic Lane-Change Maneuvers", Transactions on Intelligent Transportation Systems, IEEE, vol. 11, no.3, 2010, pp.607-616. http://dx.doi.org/10.1109/TITS.2010.2049353

[25]Broadhurst, A. E., Baker, S. and Kanade, T., "Monte Carlo Road Safety Reasoning”, Proceedings of the IEEE International Symposium on Intelligent Vehicles, 2005, pp.319-324. http://dx.doi.org/10.1109/IVS.2005.1505122

[26]Xu, G., Liu, L.and Song, Z., "Dynamic Modeling of Driver Control Strategy of Lane-Change Behavior and Trajectory Planning for Collision Prediction", Transactions on Intelligent Transportation Systems, IEEE, no.99, 2012, pp. 1-18. http://dx.doi.org/10.1109/TITS.2012.2187447

[27]Doshi, A. and Trivedi, M. M., "On the Roles of Eye Gaze and Head Dynamics in Predicting Driver's Intent to Change Lanes", Transactions on Intelligent Transportation Systems, IEEE, vol.10, no.3, 2009, pp.453-462. http://dx.doi.org/10.1109/TITS.2009.2026675

[28]Morris, B., Doshi, A. and Trivedi, M. M., "Lane Change Intent Prediction for Driver Assistance: On-Road Design and Evaluation”, Proceedings of the IEEE International Symposium on Intelligent Vehicles, 2011, pp. 895-901. http://dx.doi.org/10.1109/IVS.2011.5940538

[29]Xu, G., Liu, L. and Song, Z., "Generating Lane-change Trajectories using the Dynamic Model of Driving Behavior", Proceedings of the IEEE International conference on Information and Automation, 2011, pp.464-469. http://dx.doi.org/10.1109/ICINFA.2011.5949037

[30]Sun, Y. and Loannon, P., “A Hand Book for Inter-Vehicle Spacing in Vehicle Following”, 1995.

[31]Errampalli, M., Okushima, M. and Akiyama, T., "Fuzzy Logic Based Lane Change Model for Microscopic Traffic Flow Simulation", In Journal of Advanced Computational Intelligence and Intelligent Informatics, vol. 12, no. 2, 2008, pp.172-181.

[32]Kanaris, A., Kosmatopoulos, Elias B. and loannou, Petros A., "Strategies and Spacing Requirements for Lane Changing and Merging in Automated Highway Systems", In IEEE Transaction on Vehicular Technology, vol. 50, no. 6, 2001, pp. 1568-1581. http://dx.doi.org/10.1109/25.966586

[33] Ghumman, U., Kunwar, F. and Benhabib, B., "Guidance-Based On-line Motion Planning for Autonomous Highway Overtaking," In International Journal on Smart Sensing and Intelligent System, vol. 1, no. 2, 2008, pp. 549-571.

[34]Naranjo, J. E., Gonzalez, C., Garcia, R. and Pedro, T. D., "Cooperative Throttle and Brake Fuzzy Control for ACC+ Stop \& Go Maneuvers", In IEEE Transactions on Vehicular Technology, vol. 56, no. 4, 2007, pp. 1623-1630. http://dx.doi.org/10.1109/TVT.2007.897632

[35]Thiemann, M., Treiber, M. and Kesting, A. "Estimating Acceleration and Lane-Changing Dynamics from Next Generation Simulation Trajectory Data", In Transportation Research Record, 2088, 2008, pp. 90-101. http://dx.doi.org/10.3141/2088-10

[36]Lu, X.-Y. and Skabardonis, A., "Freeway Traffic Shockwave Analysis: Exploring the 
NGSIM Trajectory Data", In $86^{\text {th }}$ Annual Meeting of Transportation Research Board, 2007, pp. 1-19.

\section{Copyright Disclaimer}

Copyright reserved by the author(s).

This article is an open-access article distributed under the terms and conditions of the Creative Commons Attribution license (http://creativecommons.org/licenses/by/3.0/). 